\title{
A welcome statement from the associate Editor-in-Chief
}

\author{
Rupert Bartsch
}

Published online: 31 August 2016

(C) Springer-Verlag Wien 2016

It is with great pleasure that I join the editorial team of memo - Magazine of European Medical Oncology - and take over the new position of the associate Editor-inChief. memo is a noteworthy journal that I have been reading with great interest for many years and which has without doubt benefitted my clinical practice. Indeed, there is one major difference between memo and the plethora of other publications in the field of oncology: Due to its timely and relevant articels covering the entire spectrum of hematology and oncology, and its series of short (but nevertheless comprehensive) reviews, memo is a journal which is actually read-and done so by a broad range of clinicians caring for cancer patients.

Thus, obviously, the experienced and professional editorial team of memo has done a great job henceforth. Is it therefore really necessary to come up with a new position, such as that of an associate Editor-inChief? Obviously, a journal such as memo can only be successful as long as we are able to motivate renowned experts to submit update articles on their respective fields, with the help of guest editors providing us with review series on congresses or special series on hot topics in oncology and last-but not least-by motivating researchers to submit original research articles to memo. Beyond doubt it is the last point which we need to focus on in the future as it is important for memo to get listed in PubMed-which is one of our great aims.

Together with the present editorial team I will try everything to build up on our mentioned strengths but also broaden the scope of memo wherever meaningfully possible in order to ensure our journal's future success without giving up our high quality standards.

\section{Rupert Bartsch, Associate EiC}

Conflict of interest R. Bartsch declares that he has no competing interests.

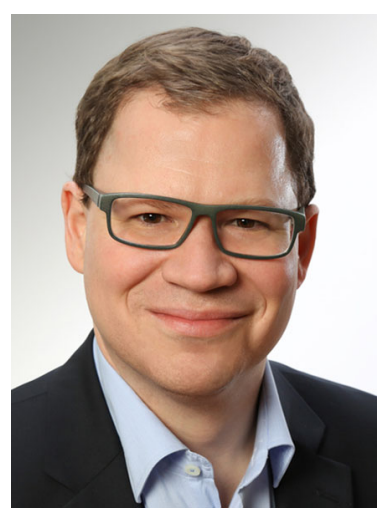

Rupert Bartsch, AssociateEiC

\footnotetext{
R. Bartsch, MD ( $\bowtie)$

Department of Medicine 1, Clinical Division of Oncology and Comprehensive Cancer Center, Medical University of Vienna, Waehringer Guertel 18-20, 1090 Vienna, Austria rupert.bartsch@meduniwien.ac.at
} 